\title{
DIABETIC AND NON-DIABETIC PATIENTS IN THE INTENSIVE CARE UNIT: MORBIDITY AND MORTALITY
}

\section{Luz, M. Felipe*, Parisi, R. Maria Cândida.}

\section{Abstract}

We conducted a prospective observational study in an intensive care unit to evaluate morbidity and mortality in diabetic patients compared to non-diabetics.

Key words: Diabetes, sepsis, intensive.

\section{Introduction}

Studies made in intensive care wards have associated stress induces hyperglycemia and mortality. Diabetes is a strong predictor of death in patients with Heart Failure. The relation between diabetes, renal disease and mortality has been related to cardiovascular disease (CVD) and hemodynamic instability during Hemodialysis. The aim of this study were to evaluate morbidity and mortality in diabetic patients compared to non-diabetics in intensive care unit.

\section{Methods}

Gender, age, total hospitalization days, number of exams (echocardiography, x-ray, computed tomography, magnetic resonance imaging), number of prescribed antibiotics, invasive procedures (dialyses catheter, nasogastric tube, hemodialysis, surgery intervention) and outcome (death, discharged to the ward or home) were analyzed.

\section{Results}

Between January and June 2017, 53 patients were studied (diabetics and non-diabetics) from an intensive unit care in a tertiary hospital.

\begin{tabular}{|l|l|}
\hline Characteristic & Frequency \\
\hline Total & 53 patients \\
\hline Gender & 31 men $(58,4 \%)$ \\
\hline DM & $19(35,8 \%)$ \\
\hline Mean Age & 57,5 years \\
\hline Mean Lenght of stay & 11,3 days \\
\hline
\end{tabular}

\begin{tabular}{|c|c|c|c|}
\hline Characteristic & $\begin{array}{l}\text { In all group } \\
\text { (53) }\end{array}$ & DM (19) & Non DM (34) \\
\hline $\begin{array}{l}\text { Diagnostic } \\
\text { Sepsis }\end{array}$ & $24(45,2 \%)$ & $12(63,1 \%)$ & $12(35,2 \%)$ \\
\hline $\begin{array}{l}\text { Diagnostic } \\
\text { CVD }\end{array}$ & $8(15 \%)$ & $2(10,5 \%)$ & $6(17,6 \%)$ \\
\hline $\begin{array}{l}\text { Diagnostic } \\
\text { Others }\end{array}$ & $21(39,6 \%)$ & $5(26,3 \%)$ & $16(47 \%)$ \\
\hline $\begin{array}{l}\text { Outcome } \\
\text { home }\end{array}$ & 26 (49\%) & $8(42,1 \%)$ & 18 (52,9\%) \\
\hline $\begin{array}{l}\text { Outcome } \\
\text { transfered }\end{array}$ & $13(24,5 \%)$ & $3(15,7 \%)$ & $10(29,4 \%)$ \\
\hline $\begin{array}{l}\text { Outcome } \\
\text { death }\end{array}$ & $14(26,4 \%)$ & $8(42,1 \%)$ & $6(17,6 \%)$ \\
\hline $\begin{array}{l}\text { Invasive } \\
\text { procedures }\end{array}$ & $43(81,1 \%)$ & $17(89,4 \%)$ & $26(76,4 \%)$ \\
\hline Hemodialysis & $13(24,5 \%)$ & $7(36,8 \%)$ & $6(17,6 \%)$ \\
\hline $\begin{array}{l}\text { Numbers of } \\
\text { ATB used }\end{array}$ & $\begin{array}{l}\bullet 0: 11,3 \% \\
\bullet 1-2: 45,2 \% \\
\bullet \geq 3: 43,3 \%\end{array}$ & $\begin{array}{l}\bullet 0: 10,5 \% \\
\bullet 1-2: 47,3 \% \\
\bullet \geq 3: 42,1 \%\end{array}$ & $\begin{array}{l}\bullet 0: 11,7 \% \\
\bullet 1-2: 44,1 \% \\
\bullet \geq 3: 44,1 \%\end{array}$ \\
\hline
\end{tabular}

\section{Conclusions}

Mortality was associated with number of antibiotics used and age. Therefore, are important points of medical attention. In addition, diabetics are presented in a more serious situation in general, need more invasive procedures and hemodialysis. We infer that the series needs to be extended, however the data point to a more fragile and more likely to unfavorable outcomes in diabetic patients.

\section{References}

1. Bauters C, Lamblin N, Mc Fadden EP, Van Belle E, Milliaire A, De Goote P. Influence of diabetes mellitus on heart failure risk and outcome. Cardiovasc Diabetol 2003, 2:1.

2. Laing SP, Swerdlow AJ, Slater SD, et al. Mortality from heart disease in cohort of 23.000 patients with insulin-treated diabetes. Diabetology 2003, 46:760-65. 